\title{
PAPERS
}

\section{Robust decentralized controller design in time domain: Equivalent subsystem approach}

\author{
Vojtech Veselý ${ }^{1}$
}

\begin{abstract}
In this paper, the original method to design of PID robust decentralized controller is obtained for linear time-invariant large-scale uncertain system. The controller design procedure performs on the subsystem level such that the closed-loop stability and performance of complex system in the frame of the designer chosen controller design procedure $\left(H_{2}, L_{2}\right.$-gain, pole placement,...) is guaranteed. The proposed method is implemented in two steps. In the first step, the required dynamic properties of the subsystems are determined so as to ensure the stability of complex system. In the second step, on the subsystem level a decentralized controller design is provided using any suitable design procedure for each subsystem.
\end{abstract}

K e y w o r d s: large-scale system, equivalent subsystem method, continuous time system, robust stability, output decentralized feedback, quadratic/parameter dependent quadratic stability

\section{Introduction}

One of the great challenges of control theory is to design robust, optimal, decentralized controller with the increasing size of dynamic uncertain large-scale system models. Such typical problems arise in the control of power, water, traffic, mechatronic systems and large real technological processes, and so on. Such complex systems are too large and too complex to be controlled. For these reasons, the complex system should to be decomposed into a number of interconnected subsystems and controlled in a decentralized fashion. In such a way, the overall plant is no longer controlled by one centralized controller but by number of independent decentralized controller [1], [2].

Rapid development of decentralized control design methods began since 1970s. For detail overview of decentralized control and their evolution, see the excellent review paper of [2]. For linear time-invariant large-scale systems, the decentralized controller design procedure has been obtained in frequency and in the time domain. In the frequency domain mainly were developed: sequential design [3], independent design method [4], and methods of equivalent subsystem approach [5]. The first two method are characterized mainly by complexity of solution and their conservatism. The last method solves the proposal of robust decentralized controller with a necessary and sufficient stability condition.

In the time domain the decentralized design procedure started with Vector Lyapunov function [6] and aggregation matrix [1]. Much progress has been made in the design of decentralized control through the use of LMI-BMI, see review article [2]. Complete complex model needs to be used when LMI-BMI approach is used when designing decentralized control. These tasks cannot be solved simply, while the systems are too large and the problem is to complex. Decentralized controllability and observability, and decentralized stabilization of complex system are given in [7] and references therein. Decentralized control for discrete-time linear systems should be found in frequency domain [5], in time domain [8] and references therein.

In this paper, a fundamentally new method of designing a decentralized controller on the subsystem level is proposed. The method consists of two steps. In the first step, such dynamic properties of subsystems are determined that the complex system should be stable. In the second step, of the task to designing a decentralized controller is to ensure the required subsystems dynamic properties. In the first step of the present method, the properties of the subsystems are determined with the necessary and sufficient conditions at which the stability of the complex system is ensured. The conservatism of the proposed method depends on the chosen design procedure when designing the decentralized controller.

We provide preliminary results that are necessary to define an equivalent subsystem and the formulation of the problem. After an equivalent subsystem and its use for the design of decentralized controller for a linear positive large-scale systems is introduced, we bring example showing the effectiveness of proposed method.

Hereafter, the following notation conditions will be adopted. Given a symmetric matrix $P=P^{\top} \in R^{n \times n}$, the inequality $P>0,(P<0)$ denotes matrix positive (negative) definiteness. $I_{n}, 0_{n}$ denotes the identity, (zero) matrix of corresponding dimensions.

\footnotetext{
${ }^{1}$ Institute of Robotics and Cybernetics, Faculty of Electrical Engineering and Information Technology, Slovak University of Technology, vojtech.vesely@stuba.sk
}

https://doi.org/10.2478/jee-2021-0046, Print (till 2015) ISSN 1335-3632, On-line ISSN 1339-309X (C) This is an open access article licensed under the Creative Commons Attribution-NonCommercial-NoDerivs License (http: //creativecommons.org/licenses/by-nc-nd/3.0/). 


\section{System description and preliminaries}

Consider the polytopic large scale continuous timeinvariant system with input and output matrices in the decentralized structure

$$
\dot{x}=A(\xi) x+B(\xi) u, \quad y=C x,
$$

where $x \in R^{n}, u \in R^{m}, y \in R^{l}$ are the state, control input, and controlled output, respectively. System matrices

$$
(A(\xi), B(\xi))=\sum_{i=1}^{N}\left(A_{i}, B_{i}\right) \xi_{i},
$$

belong to a polytopic uncertainty domain. For the uncertainty $\xi \in \Omega_{\xi}$ holds

$$
\Omega_{\xi}=\left\{\xi_{i} \geq 0, i=1,2, \ldots, N, \sum_{i=1}^{N} \xi_{i}=1, \sum_{i=1}^{N} \dot{\xi}_{i}=0\right\} .
$$

Entries of matrices $A_{i}, B_{i}, C$ are constant and last two matrices need to have in decentralized structure.

$$
\begin{gathered}
A_{i}=\left[\begin{array}{ccc}
A_{i 11} & \ldots & A_{i 1 m} \\
\ldots & \ldots & \ldots \\
A_{i m} & \ldots & A_{i m m}
\end{array}\right] \in R^{n \times n} \\
B_{i}=\operatorname{blockdiag}\left[B_{i 1} \ldots B_{i m}\right] \in R^{n \times m} \\
C=\operatorname{blockdiag}\left[C_{1} \ldots C_{m}\right] \in R^{l \times n} \quad i=1,2, \ldots, N
\end{gathered}
$$

Assume that complex system (1) is centralized controllable, observable and there are no unstable fixed modes $[9],[10],[11]$.

The system (1) can be formally decomposed to subsystems in different ways. In this paper, the division of the above matrices into sub-matrices follows from inherent properties of complex large-scale systems. Non-overlaping structure [12] will be used in the following text. The problem studied in this paper is to design of a robust PI, (PID) decentralized controller for every non-overlapping subsystem level with decentralized control algorithm

$$
\begin{aligned}
& u_{j}=k p_{j} C_{j} x_{j}+k i_{j} C_{j} \int_{t_{o}}^{\infty} x_{j} d \tau+k d_{j} C_{j} \dot{x}_{j}, \\
& j=1,2, \ldots, m,
\end{aligned}
$$

such that proposed robust controller guarantee the closedloop stability and performance of the subsystem and complex uncertain system with designer defined performance. Note that, all decentralized controller design procedure performed on the subsystem level.

\subsection{Some preliminaries}

Lemma $1,[13]$. If

$$
G=c H+\varrho I_{n},
$$

holds for scalars $c, \varrho \in R$ and identity matrix $I_{n} \in R^{n \times n}$ then the eigenvalues of matrix $G$ are to the form $\lambda_{k}=$ $c \alpha_{k}+\varrho$ where $\alpha_{k}$ is the eigenvalue of matrix $H, k=$ $1,2, \ldots, n$
Definition 1, [14]. If $G \in R^{n \times n}$, its field of values is $F(G)=\left\{v^{\top} G v, v \in C^{n}, v^{\top} v=1\right\}$.

Lemma 2. If $\lambda$ is an eigenvalue of sum of two symmetric matrices $G+H$, then $\lambda \in F(G)+F(H)$ where $F($.$) is$ the field of values of the corresponding matrix.

From Lyapunov stability theory one obtain

Lemma 3. The sum of two matrices $G+H \in R^{n \times n}$ is stable if and only if there positive definite Lyapunov matrix $P>0$ exists such that the Lyapunov inequality

$$
(G+H)^{\top} P+P(G+H) \leq 0,
$$

holds.

Definition 2. Let matrix $E=e_{i j} n \times n$ be the structured perturbation matrix of system (1) where $e_{i j}=1$, if between $i$ and $j$ subsystems an interaction connection exists, $e_{i j}=0$, if between $i$ and $j$ there is no connection.

Definition 3. A complex system (1) is connective stable if it is stable for all possible entries of matrix $E=e_{i j}$ or the system is connective stable if an interaction becomes decoupled or perturbed $\left(0<e_{i j}<1\right)$.

\section{Main results}

\subsection{Equivalent subsystem approach}

In this section, the original results to design of robust PI, (PID) decentralized controller using a Equivalent subsystem approach in time domain are obtained. Equivalent subsystem is an auxiliary subsystem to serve for design of the decentralized controller which guaranteed the stability and performance of the closed-loop subsystem diagonal matrix $A_{d}$ and complex uncertain system. Designed decentralized controller guarantee for the closedloop uncertain system the quadratic/parameter dependent quadratic stability and the performance defined by the designer. Controller design procedure performs on the subsystem level. Let's split uncertain complex system (1) to the form

$$
\dot{x}=(A d(\xi)+A m(\xi)) x+B(\xi) u ; y=C x,
$$

or for $i$-th vertex of polytope

$$
\begin{aligned}
& \dot{x}=\left(A d_{i}+A m_{i}\right) x+B_{i} u ; y=C x, \\
& u=\operatorname{blockdiag}\left\{u_{1}, u_{2}, \ldots u_{m}\right\}, i=1,2, \ldots, N,
\end{aligned}
$$

where $A d(\xi)$ is the block diagonal part of matrix $A(\xi)$ and $A m(\xi)=A(\xi)-A d(\xi)$ is diagonal off part of system (1). 
Theorem 1. Let us have two matrices $A d_{i}, A m_{i}$ with constant entries. The following sum $D_{i}=A d_{i}+I \alpha+A m_{i}$ is stable for some $\alpha$ if positive definite matrix $P_{i} \in R^{n \times n}$ exists, such that the following inequality holds

$$
\left(A d_{i}+A m_{i}\right)^{\top} P_{i}+P_{i}\left(A d_{i}+A m_{i}\right)+2 \alpha P_{i}<0 .
$$

\section{P r o of . Due to Lemma 1 one obtains}

$$
\left(A d_{i}+I \alpha+A m_{i}\right)^{\top} P_{i}+P_{i}\left(A d i+I \alpha+A m_{i}\right)<0 .
$$

After small manipulation of (12) one obtain (11), which proofs the sufficient stability condition. Let us assume that matrix $D_{i}$ is stable, then using Lyapunov stability theory there is a positive definite matrix $P_{i}$ exists such that inequality (11) holds, which proofs the theorem.

Consider the uncertain polytopic system in the form $\dot{x}=A(\xi) x=\sum_{i=0}^{N}\left(\left(A d_{i}+I \alpha+A m_{i}\right) \xi_{i}\right) x$. The stability of such complex uncertain system after small modification of [15] is given in the following.

Lemma 4. The linear uncertain polytopic system $A(\xi)$ is stable if matrices $G, H \in R^{n \times n}$, parameter $\alpha$ and symmetric positive definite matrix $P_{i}, i=1,2, \ldots, N$ exist, such that the following inequality holds

$$
\begin{gathered}
{\left[\begin{array}{cc}
D_{i}^{\top} H^{\top}+H\left(A d_{i}+I \alpha+A m_{i}\right) & * \\
P_{i}-H^{\top}+G^{\top}\left(A d_{i}+I \alpha+A m_{i}\right) & -G-G^{\top}
\end{array}\right]<0} \\
i=1,2, \ldots, N .
\end{gathered}
$$

From (10) or (12) one obtains the parameter $\alpha$. If obtained parameter $\alpha \geq 0$, the complex system is stable. In this case decentralized controller should be designed such that for all closed-loop $j-t h$ subsystems eigenvalues next inequality holds

$$
\begin{aligned}
& \lambda_{k}\left(\left(A d_{i}+B_{i} * P I C\right)\right)<\lambda_{k}\left(A d_{i}+\alpha I\right), \\
& k=1,2, \ldots, d,
\end{aligned}
$$

where: $P I C=$ PI-controller. The decentralized controller should not make dynamics of the corresponding subsystem worse than the without controller. If $\alpha<0$ is obtained then the complex system is unstable. To with cope this problem of the decentralized controller design the following auxiliary equivalent subsystem matrix was introduced, chose $\beta=|\alpha|+\delta$ where $\delta \geq 0$ is small tuning parameter (for the first step $\delta=0$ ).

Definition 4. Equivalent subsystem is defined as

$$
A e_{i}=A d_{i}+I \beta .
$$

Let us assume, static output feedback with gain matrix $K,(P, P I)$ controller which guarantees the stability of closed-loop equivalent subsystems. Then the following corollaries hold
Corollary 1. Closed-loop complex system is stable if

- $A e_{i}+B_{i} K_{i} C, \quad i=1,2, \ldots, N$ is stable or all closedloop subsystems are stable

- It is sufficient if $\lambda_{k}\left(A d_{i}+B_{i} K_{i} C\right)<\lambda_{k}\left(A d_{i}+I \alpha\right), i=$ $1,2, \ldots, N, k=1,2, \ldots, d$ holds for all dominant eigenvalues. Otherwise, the stability of complex system needs to be checked, using (12) for uncertain system and system without uncertainty by (11). For the obtained $\alpha \geq 0$ the complex system is stable.

- Assume that complex system (1) is centralized controllable, observable and there is no unstable fixed mode.

Summarizing the results, the following decentralized controller design procedure has been obtained.

Algorithm for decentralized controller design

Assume that complex system is decentralized controllable and observable, [7] and there no unstable fixed mode.

- In the first step the value of $\alpha$ is calculated using for classical MIMO systems (11) or for uncertain polytopic system, (12). If $\alpha \geq 0$ (the complex system is stable), decentralized controllers are designed for all subsystems using any controller design procedure such that for the following dominant inequalities hold $E C L\left(A d_{i j}+B_{i j} * C * C(j)\right)_{k}<E O L\left(A d_{i j}+I_{j} \alpha\right)_{k}$, $i=1,2, \ldots, N ; j=1,2, \ldots, m, k=1,2, \ldots, d_{j}$;. Where: $C=$ controller and $E C L=$ eig-closed-loop, $E O L=$ eig-open-loop. Note that, $d_{j}, d$ in this time determined by experimental way. The stability of a complex linear and nonlinear system can be determined using an aggregation matrix, [1], [16]. If the system is stable and the diagonal elements of the aggregation matrix are not increased by decentralized controllers, the stability of the system will not be disturbed. This paper specifies the result achieved using the aggregation matrix.

- Complex system is unstable, $\alpha<0$. Define the equivalent subsystem using (13) where for the first step one puts $\delta=\left(\begin{array}{ll}0 & 0.1\end{array}\right)$. Design a robust decentralized controller on the equivalent subsystem level using any designed method such that all closed-loop (equivalent subsystems+decentralized controllers) should be stable. The complex system should be stable if for all closed-loop subsystem eigenvalues the following hold $E C L\left(A d_{i j}+B_{i j} * C * C(j)\right)_{k}<E O L\left(A d_{i j}+\alpha * I_{j}\right)_{k}$, $k=1,2, \ldots, d_{j}$. Otherwise, increase $\delta[1],[16]$ and repeat the calculation from the second step. Check the closed-loop stability of the complex system with stable subsystems by calculation of new $\alpha$. If $\alpha \geq 0$ than the complex system is stable.Note that if I-part of the decentralized controller is used for all subsystems then $m$ new subsystems states are introduced. In this case the interaction matrix $A m$ need to be modified by zero elements.

- If new calculated $\alpha<0$ and the complex system could not be stabilized increasing $\delta$, then there is fixed unstable mode in the complex system. See characterization of fixed modes and criteria for its testing in [10]. 


\section{Example}

The example is rather academic. The goal is given as follows. Design a robust decentralized PI controller for the given positive uncertain polytopic system.

\subsection{Robust decentralized controller design for positive systems}

Many real plant models belong to the class of positive systems. In this subsection, the controller design procedure will be obtained for positive systems in the frame of $\mathrm{H}_{2}$.

Definition 5. A linear uncertain system (1) is said to be positive if both state and output are non negative for all non negative initial states and input.

Definition6. The real matrix $D\left\{d_{i j}\right\}$ is Metzler if it has non negative off-diagonal entries, $d_{i j} \geq 0, i \neq j$. Metzler matrix is stable if and only if all its eigenvalues lay in the left half of the complex plane

For more detail about positive systems, see excellent tutorials [17] and [18].

For i-th $x$ vertex and j-th equivalent subsystem of complex system, one obtains the following subsystem model

$$
\dot{x}_{j}=A e_{i j} x_{j}+B_{i j} u_{j} ; y_{j}=C_{j} x_{j} .
$$

To obtain static output feedback with PI controller in the subsystem model,the number of state variables needs to be increased [19]. In the decentralized control algorithm (5), integral term can be included into the state vector defining new state as $z_{j}=\int_{t o}^{\infty} y_{j}(\tau) d \tau$, e.i. $\dot{z}_{j}=y_{j}=$ $C_{j} x_{j}$. PID control algorithm is in the form

$$
\begin{gathered}
u_{j}=\left[\begin{array}{ll}
k p_{j} C_{j} & k i_{j}
\end{array}\right]\left[\begin{array}{c}
x_{j} \\
z_{j}
\end{array}\right]+\left[\begin{array}{ll}
k d_{j} C_{j} & 0
\end{array}\right]\left[\begin{array}{c}
\dot{x}_{j} \\
\dot{z}_{j}
\end{array}\right], \\
j=1,2, \ldots, m,
\end{gathered}
$$

or $u_{j}=K_{j} x_{n j}+K_{D} \dot{x}_{n j}$. Closed-loop system for PI controller is described as

$$
\dot{x}_{n j}=\left[\begin{array}{cc}
A e_{i j}+B_{i j} k p_{j} C_{j} & B_{i j} k i_{j} \\
C_{j} & 0
\end{array}\right] x_{n j} .
$$

From the equation (16) follows that the following conditions need to be satisfied to guarantee the closed-loop matrix Metzler properties and stability with PI controller:

- The matrix $A e_{i j}+B_{i j} k p_{j} C_{j}$ is a stable Metzler matrix.

- The matrix $B_{i j} k i_{j}$ needs to have positive entries or integral part of controller needs to have positive feedback.

The second point is crucial problem to guarantee the stability of (16). This paper proposes using of the approximate I part of the controller to get over this problem, as follows

$$
\dot{z}_{j}=y_{j}-\gamma z_{j}
$$

where $\gamma \geq 0$ is tuning parameter, $1 / \gamma>(3-5) T s$ where Ts is maximal value of system time constant. Note that, if $\gamma=0$ the proposed decentralized control design procedure could be used for classical systems. The closedloop (16) for i-th vertex is changes to

$$
\dot{x}_{n}=A c_{i} x_{n} \quad A c_{i}=\left[\begin{array}{cc}
A e_{i}+B_{i} k p C & B_{i} k i \\
C & -I \gamma
\end{array}\right] \in R^{e \times e},
$$

where matrices $A e_{i}, B_{i}, k p, k i$ are block diagonal matrices with corresponding subsystem matrices. All uncertain closed-loop complex plant is

$\dot{x}_{n}=(A c(\xi)+\bar{A} m(\xi)) x_{n} ; \quad \bar{A} m(\xi)=\left[\begin{array}{cc}A m(\xi) & 0 \\ 0 & 0\end{array}\right] \in R^{e \times e}$

For polytopic equivalent subsystem, one should choose the Lyapunov function as

$$
V(\xi)=x_{n}^{\top} P(\xi) x_{n} ; \quad P(\xi)=\sum_{i=1}^{N} P_{i} \xi_{i}
$$

The first time derivative of Lyapunov function gives

$$
\dot{V}(.)=v_{1}^{\top}\left[\begin{array}{cc}
P(\dot{\xi}) & P(\xi) \\
P(\xi) & 0
\end{array}\right] v_{1} ; \quad v_{1}^{\top}=\left[\begin{array}{ll}
x_{n}^{\top} & \dot{x}_{n}^{\top}
\end{array}\right] .
$$

To reduce the conservativeness and split the subsystem matrices to a Lyapunov matrix the following two auxiliary matrices $N_{1}, N_{2}$ are introduced

$$
v_{1}^{\top}\left[\begin{array}{l}
2 N_{1}^{\top} \\
2 N_{2}^{\top}
\end{array}\right][A c(\xi) \quad-I] v_{1}=0 .
$$

Summarizing (21) and (22) one obtains the time derivative of Lyapunov function for all equivalent subsystems

$$
\begin{aligned}
& \frac{d V(\xi)}{d t}= \\
& =v_{1}^{\top}\left[\begin{array}{cc}
N_{1}^{\top} A c(\xi)+A c(\xi)^{\top} N_{1} & -N_{1}^{\top}+A c(\xi) N_{2} \\
-N_{1}+N_{2}^{\top} A c(\xi) & -N_{2}^{\top}-N_{2}
\end{array}\right] v_{1} .
\end{aligned}
$$

Equation(23) implies that if $\frac{d V(\xi}{d t}$ is negative the closed-loop system is stable. There are many ways to obtain PI controller design procedure for an equivalent subsystem. Some of them are: $H_{2}$-approach, $H_{\text {inf }}$, regional pole placement approach, etc. There is only one important constraint to guarantee the stability of complex plane given by Corollaries 1 item 2. In this paper the $\mathrm{H}_{2}$ approach is used with LQSR (state, derivative of state and input) quadratic performance

$$
\begin{aligned}
& J s=\int_{t o}^{\top} J d t ; \quad J=x_{n}^{\top} Q x_{n}+\dot{x}_{n}^{\top} S \dot{x}_{n}+u^{\top} R u, \\
& j=1,2, \ldots, m,
\end{aligned}
$$

where $Q, S \in R^{e \times e} \geq 0, R>0$ are matrices.

In the next the Bellman-Lyapunov equation is used to obtain robust PI controller design procedure. 
Lemma 5, [20]. Consider the uncertain positive system $\dot{x}_{n}=A c(\xi) x_{n}$ with decentralized structure. Decentralized control algorithm (15) for PI controller belongs to the class of guaranteed cost control for closed-loop system if and only if there is a positive definite Lyapunov function existing and $\varepsilon \geq 0$ such that following condition holds:

$$
B e=\max _{u_{j}}\left\{\frac{d V(\xi)}{d t}+J\right\}=-\varepsilon x_{n}^{\top} x_{n} .
$$

Substitution (23) and (24) to (25) leads to obtaining of the robust decentralized controller design procedure. Designed controller ensures guaranteed cost with optimal value of quadratic cost function (24). In the frame of $\mathrm{H}_{2}$ the designed controller needs satisfy the following inequality

$$
\begin{gathered}
B e=v_{1}^{\top} W(\xi) v_{1}<0 ; \quad W(\xi)=\sum_{i=1}^{N} W_{i} \xi_{i}, \\
W_{i}=\left\{w_{k l i}\right\}_{2 \times 2} ; \quad i=1,2, \ldots, N .
\end{gathered}
$$

Note, that for the Lyapunov function chosen by the designer in (26) conditions "if and only if" may be reduce to "if".

$$
\begin{aligned}
& \text { and } \\
& \begin{array}{c}
w_{11 i}=N_{1}^{\top} A c_{i}+A c_{i}^{\top} N_{1}+Q+K^{\top} R K+\sum_{i=1}^{N} P_{i} \dot{\xi}_{i}, \\
w_{12 i}=-N_{1}^{\top}+N_{2}^{\top} A c_{i}+P_{i} ; \quad w_{21 i}=w_{12 i}^{\top} \\
w_{22 i}=-N_{2}^{\top}-N_{2}+S,
\end{array}
\end{aligned}
$$

where $K=[k p C k i]$.

Optimal control is obtained if $\operatorname{Tr}\left(P_{i}\right) \rightarrow \min , i=$ $1,2, \ldots, N$. The inequality (26) defines the robust decentralized controller design procedure for positive system in the frame of $\mathrm{H}_{2}$.

Theorem 2. The uncertain polytopic positive subsystem (14) is robust parameter dependent quadratically stable/quadratic stable with guaranteed cost if for the given performance matrices $Q, S, R$ and bounds $\xi \in \Omega_{\xi}$ two auxiliary matrices $N_{1}, N_{2}$, symmetric positive definite matrix $P_{i}>0$ and decentralized controller gain matrices $k p, k i$ exist such that inequality (26) holds. If the conditions of Corollaries 1 are met the complex positive system is stable and satisfies conditions of connective stability of a complex system.

P r o o f . Sufficient stability conditions of above Theorem results from the previous consideration, eqs. (18)(25) and Corollaries 1.

Note that all matrices in (26) are all block diagonal matrices and all decentralized controller design procedure perform on the subsystem level. For matrix $A c_{i}$ and $K_{j}$ holds

$$
\begin{gathered}
A c_{i}=\text { blockdiag }\left\{\left[\begin{array}{cc}
A e_{i j}+B_{i j} k p_{j} C_{j} & B_{i j} k i_{j} \\
C_{j} & -I_{j} \gamma
\end{array}\right]\right\}, \\
i=1,2, . ., N, j=1,2, \ldots, m, K_{j}=\left[\begin{array}{ll}
k p_{j} C j & k i_{j}
\end{array}\right] .
\end{gathered}
$$

\subsection{Model and parameters}

State and output feedback (for comparison) decentralized controller need to be designed for the given positive uncertain polytopic system with two vertices and two subsystems. $\mathrm{H}_{2}$ performance with quadratic cost function defining the quality of the complex system is given as follows, (24) $Q_{j}=q I, q=5 \cdot 10^{-3}, S_{j}=s I, s=10^{-5}, R_{j}=$ $r I_{m}, r=1, j=1,2$. The system matrices are as follows: $X(\xi)=X_{1} \xi_{1}+X_{2} \xi_{2} ; X=A, B$

The first vertex, $i=1$

$$
\begin{gathered}
A_{1}=\left[\begin{array}{cccc}
-2 & 0.2 & 0.2 & 0.1 \\
0.35 & -0.005 & 0.15 & 0.037 \\
0.08 & 0.22 & -0.5 & 0.16 \\
0.03 & 0.16 & 0.18 & -0.25
\end{array}\right], \\
B_{1}^{\top}=\left[\begin{array}{ll}
0 & 1
\end{array}\right] .
\end{gathered}
$$

The second vertex, $i=2$

$$
\begin{gathered}
A_{2}=\left[\begin{array}{cccc}
-1.8 & 0.15 & 0.15 & 0.03 \\
0.22 & -0.25 & 0.12 & 0.22 \\
0.072 & 0.22 & -0.4 & 0.1 \\
0.01 & 0.11 & 0.2 & -0.3
\end{array}\right], \\
B_{1}^{\top}=\left[\begin{array}{ll}
0 & 1
\end{array}\right] .
\end{gathered}
$$

Output matrix for state and output feedback

State Feedback: $C=\left[\begin{array}{ll}1 & 0 \\ 0 & 1\end{array}\right]$,

Output Feedback: $C=\left[\begin{array}{ll}0 & 1\end{array}\right]$.

\section{Robust decentralized state feedback controller}

In the first step, one calculates the value of $\alpha$ from Lemma (4) and (12). The value $\alpha=-0.0533$ is obtained. Complex uncertain system is unstable. In order to guarantee the stability of the complex system the proposed state feedback controller should shift subsystem's eigenvalues to the left by the value $\alpha$. The tuning parameter is $\delta=0.05$. Using $H_{2}$ performance the obtained state feedback controller (26) for the first and second subsystems are given as follows:

The first subsystem

$$
k p_{11}=1.588, \quad k p_{12}=-16.249 .
$$

\section{The second subsystem}

$$
k p_{21}=1.6179, \quad k p_{22}=-16.2488 .
$$

For comparison, eigenvalues of two subsystems $\operatorname{Eig}(i, j), i=$ 1,$2 ; j=1,2$ without and with state feedback controller are given below:

$$
\operatorname{Eig}(1,1)=\{-2.0345,0.0295\},
$$




$$
\begin{gathered}
\operatorname{Eig}(1,1)(\text { control })=\{-1.9729,-16.2812\} \\
\text { Eig }(1,2)=\{-0.5858,-0.1642\} \\
\text { Eig }(1,2)(\text { control })=\{-0.482,-16.5178\} \\
\text { Eig }(2,1)=\{-1.821,-0.229\} \\
\operatorname{Eig}(2,1)(\text { control })=\{-1.7819,-18.142\} \\
\text { Eig }(2,2)=\{-0.5-0.2\} \\
\operatorname{Eig}(2,2)(\text { control })=\{-0.3990,-18.1899\}
\end{gathered}
$$

The obtained eigenvalues Eig $(i, j)$ (control) clearly show that Corollary 1 . holds and the complex system is stable, which proofs the calculated values of closed-loop eigenvalues for $i=1$. The state feedback closed-loop matrix is

$$
A_{c 1}=\left[\begin{array}{cccc}
-2 & 0.2 & 0.2 & 0.1 \\
1.938 & -16.254 & 0.15 & .037 \\
0.08 & .22 & -0.5 & .16 \\
0.03 & 0.16 & 1.798 & -16.499
\end{array}\right] \in R^{M}
$$

where $\boldsymbol{M}=$ Metzler matrix, and its eigenvalues are

$$
\operatorname{Eig}\left(A_{c 1}\right)=\{-1.9882,-0.4647,-16.2708,-16.5292\}
$$

\section{Robust decentralized output feedback PI controller}

The goal is to design two PI decentralized static output feedback controllers such that the stability of complex system is guaranteed. Assume, that decentralized controllable and observable conditions for system (1) [7], hold.

For tuning parameters $\delta=0.05, \gamma=0.1$ and system parameters given above the decentralized PI controller parameters are as follows:

$$
\begin{aligned}
& \text { The first subsystem, } j=1 \\
& \qquad R_{1}(s)=-22.5252+\frac{2.22}{s+\gamma} .
\end{aligned}
$$

The first subsystem, $j=2$

$$
R_{2}(s)=-22.4904+\frac{2.2339}{s+\gamma} .
$$

Note, that sign (-) means the negative feedback. In this case, to guarantee the Metzler properties of a closedloop system (17), integral part of controller should have positive feedback. Closed-loop eigenvalues for $i=2$, and two cases $\delta=0.05$ and $\delta=0.2$ given below

$$
\begin{aligned}
& \delta=0.05 \\
& E I G\left(A_{c 2}\right)=\{-24.9796,-25.2865,-1.8066 \\
& -0.3907,-0.0009,-0.0028\} \\
& \delta=0.2 \\
& E I G\left(A_{c 2}\right)=\{-25.017,-25.3244,-1.8066 \\
& -0.3907,-0.0016,-0.0035\}
\end{aligned}
$$

Robust decentralized output feedback P-controller

Closed-loop eigenvalues for $\delta=0.2 ; i=2$, designed P-controller for both subsystems with gains $R_{1}(s)=$ $-16.2526 ; \quad R_{2}(s)=-16.299$ are as follows

$$
A_{c 2} \in R^{M}
$$

$$
\operatorname{EIG}\left(A_{c 2}\right)=\{-1.806,-0.3893,-17.9983,-18.308\} .
$$

If we compare above three examples, we can clearly state that for the case when the Metzler conditions are met the closed-loop system with robust state decentralized feedback controller has the best dynamic properties. We also have got relatively good results with the decentralized P- controller. The approximate PI controller will run very slowly.

\section{Conclusion}

In the present paper, the original method of designing a robust decentralized controller for linear uncertain and complex system is given. Task solution is implemented in two steps. The desired properties of decentralized regulators are calculated in the first step such that to ensure the stability of the complex system and the required quality of subsystems control. In the second step, the controller is proposed so, that the requirements on the decentralized controllers have been met at a maximum extent. The second step analytical solution is not fully resolved so far.

\section{Acknowledgment}

This work has been supported by Grant VEGA 1/075 4 /19 of the Slovak Scientific Grant Agency.

\section{REFERENCES}

[1] D. D. Siljak, Large-scale Dynamic Systems, Stability and Structure, North-Holland, New York, 1978.

[2] L. Bakule, "Decentralized Control: An Overview, Annual Reviews in Control, vol. 32, pp. 87-98, 2008.

[3] M. Hovd and S. Skogestad, "Sequantial design of decentralized controllers", Automatica, vol. 30, no. 10, pp. 1601-1607, 1994.

[4] M. Hovd and S. Skogestad, "Improved independent design of robust decentralized control", Journal of process Control, vol. 3, no. 1, pp. 43-51, 1993.

[5] A. Kozakova, V. Vesely, and V. Kucera, "Robust Decentralized Controller Design Based on Equivalent Subsystems", Automatica, vol. 107, pp. 29-35, 2019.

[6] V. M. Matrosov, "On the theory of stability motion", Prikladnaja matematika i Mekhanika, no. 26, pp. 992-1002, 1962, (in Russian).

[7] W. Naiqi, L. Renhou and H. Baosheng, "Decentralized controllability and observability, and decentralized stabilization", Proc. of the 1988 IEEE Int. Conf. on Systems, Man, and Cybernetics, DOI: 10. 1109/ICSMC. 1988. 754370.

[8] J. Xu, L. Xu, L. Xie, and H. Zhang, "Decentralized Control for linear systems with multiple input chanels", Science China Information Sciences, vol. 62, no. 1, Art. N. 52209, 2019.

[9] H. Seraji, "On fixed modes in decentralized control systems", Inter. J. of Control, vol. 35, no. 5, pp. 775-784, 1982. 
[10] Z. Gong and M. Aldeen, "On the characterization of fixed modes in decentralized control", IEEE Trans. on AC, vol. 37, no. 7, pp. 1046-1050, 1992.

[11] Shih-Ho Wang and E. Davison, "On the stabilization of decentralized Control Systems", IEEE Trans. on AC vol. 18, no. 5, pp. 473-478, 1973.

[12] Xue-Bo Chen and S. S. Stankovic, "Decomposition and decentralized control of Systems with multi-overlaping structure", $\mathrm{Au}$ tomatika, vol. 41, 2005, pp. 1765-1772.

[13] T. S. Shores, "Applied linear Algebra and Matrix Analysis, Springer-Verlag, New York, 2007.

[14] H. Wielandt, "On the eigenvalues of $A+B$ and AB, Journal of research of the National Burrea of Standarts", Mathematical Sciences, vol. 77B, no. 1-2, January-June, pp. 61-63, 1973.

[15] D. Peaucelle, D. Alzelier, O. Bachelier, and J. Bernussou, "A new robust D stability Condition for real Convex polytopic Uncertainty", Systems and Control Letters, vol. 40, pp. 21-30, 2000.

[16] V. Vesely, "Large -scale dynamic system stabilization using the principle of dominant subsystem approach", Kybernetika, vol. 24, no. 1, pp. 48-61, 1993.

[17] A. Rantzer and M. E. Volcher, "A tutorial on positive systems", Conf. on Decis. and Control, IEEE, 2018.

[18] C. Wang and T. Huang, "Static output feedback control for positive linear continuous-time systems", Int. J. of Robust and Nonlinear Control, vol. 23, pp. 1537-1544, 2013.
[19] V. Vesely, and D. Rosinova, "Robust PID-PSD controller design: BMI approach", Asian Journal of Control, vol. 15, no. 2, pp. 469-478, 2013.

[20] V. M. Kuncevich and M. M. Lycak, Control system design using Lyapunov function approach, Nauka, Moscow, 1977 (in Russian).

Vojtech Veselý was born in Velké Kapušany, Slovakia in 1940. He received MSc degree in Electrical Engineering from the Leningrad Electrical Engineering Institute, St. Peterburg, Russia, in 1964, PhD and DSc degrees from the Slovak University of Technology, Bratislava, Slovakia, in 1971 and 1985, respectively. Since 1964 he has been with the Department of Automatic Control Systems, STU FEI in Bratislava. Since 1986 he has been a full professor. His research interests include the areas of power system control, decentralized control of large-scale systems, robust control, predictive control and optimization. He is author or coauthor of more than 300 scientific papers.

Received 8 May 2021 